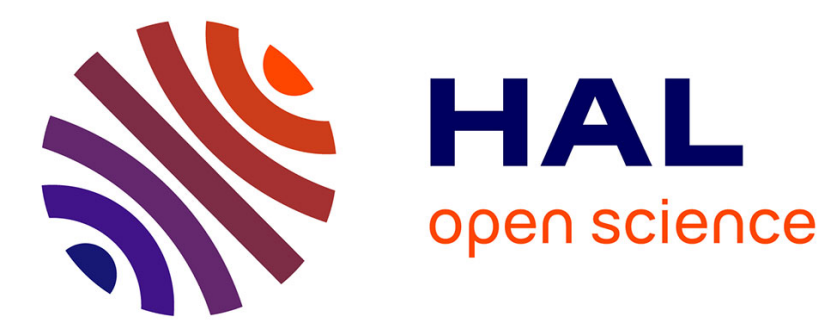

\title{
Modeling the electrical activity of a neuron by a continuous and piecewise affine hybrid system
}

Jean-Guillaume Dumas, Aude Rondepierre

\section{To cite this version:}

Jean-Guillaume Dumas, Aude Rondepierre. Modeling the electrical activity of a neuron by a continuous and piecewise affine hybrid system. Hybrid Systems: Computation and Control, Apr 2003, Prague, Czech Republic. pp.156-171, 10.1007/3-540-36580-X_14 . hal-01807138

\section{HAL Id: hal-01807138 \\ https://hal.science/hal-01807138}

Submitted on 4 Jun 2018

HAL is a multi-disciplinary open access archive for the deposit and dissemination of scientific research documents, whether they are published or not. The documents may come from teaching and research institutions in France or abroad, or from public or private research centers.
L'archive ouverte pluridisciplinaire HAL, est destinée au dépôt et à la diffusion de documents scientifiques de niveau recherche, publiés ou non, émanant des établissements d'enseignement et de recherche français ou étrangers, des laboratoires publics ou privés. 


\title{
Modeling the electrical activity of a neuron by a continuous and piecewise affine hybrid system
}

\author{
Jean-Guillaume Dumas and Aude Rondepierre \\ Laboratoire de Modélisation et Calcul \\ Tour IRMA - BP 53, 38041 Grenoble cedex 9. France \\ $\{$ Jean-Guillaume.Dumas, Aude.Rondepierre\}@imag.fr \\ www-lmc.imag.fr/lmc-mosaic/ \{Jean-Guillaume.Dumas,Aude.Rondepierre\}
}

\begin{abstract}
A hybrid system is proposed to model the electrical potential emitted by a neuron as a response to an externally applied DC current. Experimentally, Hodgkin and Huxley built a four-dimensional and nonlinear dynamical system to simulate this activity. Our idea is to use a new continuous and piecewise affine approximation as a hybrid model of the Hodgkin-Huxley dynamic. Our new model reproduces the HodgkinHuxley features with good accuracy (e.g. including the fact that the incoming current intensity is a bifurcation parameter), and, moreover, still allows an analytic computation of its solutions.
\end{abstract}

\section{Introduction}

Neurons communicate with each other by generating electrical signals, called action potentials. Action potentials are the result of currents that pass through ion channels in the cell membrane. Each neuron integrates the incoming signals and, when the stimulation reaches a certain threshold, an output signal is generated and delivered. This paper deals with the dynamics of the membrane potential of a single neuron under a certain stimulus.

As a result of their experiments on a giant squid nerve fiber, Hodgkin and Huxley proposed a first model describing the dynamics of the action potentials [9]. The main idea is that because of active ion transport (mainly sodium, potassium and chloride) through the cell membrane, this membrane acts as a capacitor. From then their measures tended to show that potassium ions can cross the membrane when four similar molecules occupy a certain region of this membrane (so that potassium conductance is considered proportional to the number of sites on the inside of the membrane which are occupied by the four molecules, $\left.g_{K} \approx \bar{g}_{K} n^{4}\right)$. Similarly, the sodium conductance is supposed to be proportional to the number of sites on the inside of the membrane which are occupied simultaneously by three activating molecules and not blocked by an inactivating molecule $\left(g_{N a} \approx \bar{g}_{N a} m^{3} h\right)$. The chloride and other ions conductance is supposed constant. From this assumptions, Hodgkin and Huxley then proposed the following dynamical system as a simplified neuron model: 
$(\mathrm{H}-\mathrm{H})\left\{\begin{array}{l}C_{M} \frac{d V}{d t}=I(t)-\bar{g}_{K} n^{4}\left(V-V_{K}\right)-\bar{g}_{N a} m^{3} h\left(V-V_{N a}\right)-\bar{g}_{l}\left(V-V_{l}\right) \\ \frac{d n}{d t}=(1-n) \cdot \alpha_{n}(V)-n \cdot \beta_{n}(V) \\ \frac{d m}{d t}=(1-m) \cdot \alpha_{m}(V)-m \cdot \beta_{m}(V) \\ \frac{d h}{d t}=(1-h) \cdot \alpha_{h}(V)-h \cdot \beta_{h}(V)\end{array}\right.$

- $V$ is the displacement of the membrane potential from its resting value

- $n$ is the proportion of the $K^{+}$activating molecules inside the membrane at time $\mathrm{t} ; 0 \leq n \leq 1$

- $m$ is the proportion of the $\mathrm{Na}^{+}$activating molecules inside the membrane at time $\mathrm{t} ; 0 \leq m \leq 1$

- $1-h$ is the proportion of the $\mathrm{Na}^{+}$inactivating molecules inside the membrane at time $\mathrm{t} ; 0 \leq h \leq 1$

- $I(t)$ is the intensity of the current applied to the neuron cell.

The $\alpha$ and $\beta$ being respectively the transfer rate constants from outside to inside and from inside to outside, the $\bar{g}_{X}$ and $V_{X}$ being respectively the conductances and resting potentials of potassium, sodium and chloride ions [11].

Despite the small dimension of the $(\mathrm{H}-\mathrm{H})$ system, the mathematical analysis is still quite complex. Therefore only numerical simulations are used and simplified models are required for a better understanding of the neuronal dynamics.

Some simplifications have been proposed [1], models like integrate-and-fire, resonate-and-fire ([13]) or even Hopfield or FitzHugh-Nagumo (see [15, §6.5] or [7, $\S 3.1]$ for further references) provide different level of relevant informations on the neuronal dynamics. For instance, these models are used to show the existence of a spike (excitable state), or of a threshold potential for the emission of a periodical state (see e.g. [7, §3.3] for more details). Unfortunately, these simplifications give only a few fully analytical results or low quality numerical approximations. Therefore some piecewise linear simplifications have been proposed by Tonnelier [16] to combine analytic results and good numerical approximations. Still there is no complete analytic analysis of the dynamics and Tonnelier's approximations suffer from a non-continuous behavior.

Our approach is to use a hybrid system modeling and to refine Tonnelier's approximation. Indeed the main advantage of an hybrid system is that it combines fully symbolic and multi-scale resolutions. On the one hand, by using continuous and linear approximations, any refinement is possible: the original system is replaced by piecewise linear parts at as many points as needed to ensure any quality of approximation. On the other hand in each resulting "cell" (corresponding to a state of the hybrid system), the system is fully linear and therefore analytically solvable. These two aspects together enable an analytic analysis of most of any system properties, whatever its complexity is.

There are many possible linearization by parts. For instance, one can build a mesh of the phase space and use multi-dimensional interpolation to define a linear approximation of the system in each cell (simplex) of the mesh, see $[4,8,2]$ for 
more details. We present a few simultations using this approximation in section 5.3. In this paper, we will instead use another idea: we linearize each equation separately by implicit representation and one-dimensional linearization on each variable, see sections 2.2 and 2.3 (using the latter on the Hodgkin-Huxley model, we actually need fewer hybrid states to prove the desired analytic properties).

We therefore propose a new continuous and piecewise affine approximation of the Hodgkin-Huxley model. We implicitly build a hybrid automaton defined by the pieces of the approximation within the phase plane of the dynamical system (see figure 6). Now, in each state, the solutions of our model are analytically computable. Moreover, simulations of this automaton show the good quality of our approximations.

In section 2, we give a reduction from the four-dimensional Hodgkin-Huxley model to a two-dimensional continuous and piecewise affine model. We next linearize both equations separately by way of an implicit representation in sections 2.2 and 2.3 and propose the associated hybrid automaton in section 3 . We then fully analyze the model dynamics in section 4 and produce some simulations and comparisons between the Hodgkin-Huxley model and our approximation in section 5 .

\section{Reduction of the Hodgkin-Huxley model}

In this section we propose a reduction from the Hodgkin-Huxley model to our piecewise affine and continuous model. We combine both FitzHugh-Nagumo [7, $\S 3.1 .1]$ and Tonnelier's approaches [16, §1.4.3] and refine them.

\subsection{From four dimensions to two dimensions}

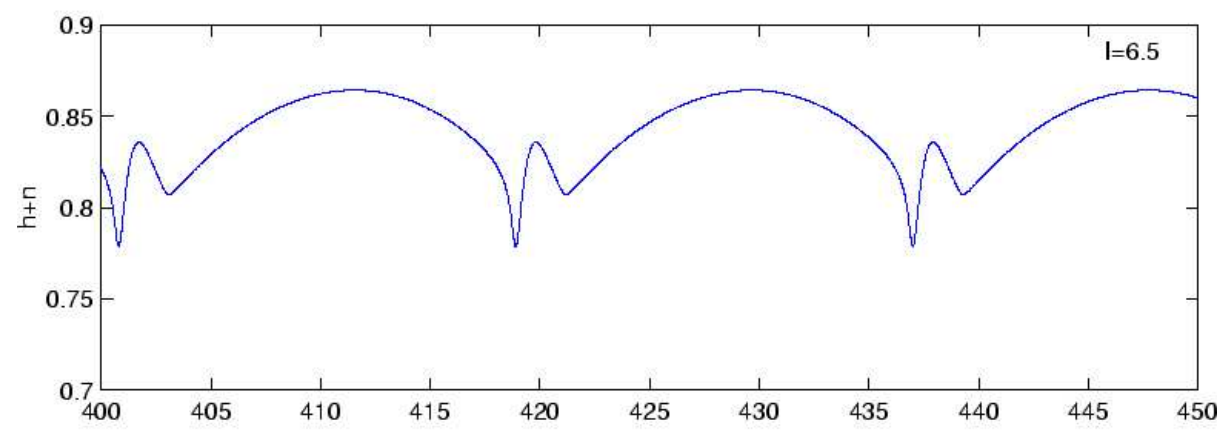

Fig. 1. Simulation of $n+h$ variation with time for a low intensity

The first step is to reduce the dimension. We first rewrite the activating or inactivating molecule equations in the form $X=-\frac{1}{\tau_{X}(V)}(X-\bar{X}(V))$. Then for 


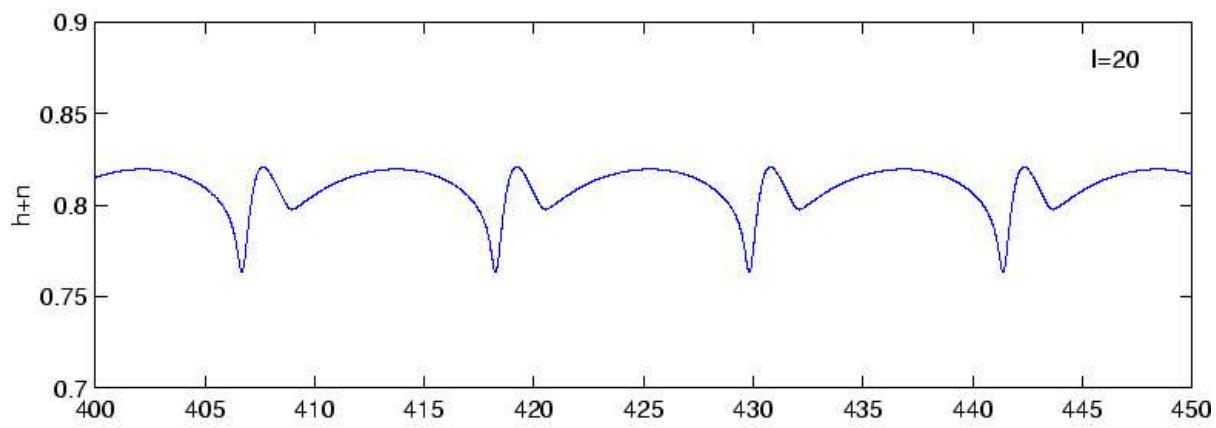

Fig. 2. Simulation of $n+h$ variation with time for a high intensity

a fixed potential $V$, the variable $X$ approaches the value $\bar{X}(V)$ (its asymptotic value) with a time constant $\tau_{X}(V)$. Experimental data shows that the variable $m$ always reaches its asymptotic value $\bar{m}(V)$ much faster than the variables $n$ and $h$ do $[7, \S 2.2 .1]$. The first hypothesis is then that the m-gate of sodium are instantaneously activated, so that we can assume $m \approx \bar{m}(V)$. Then, FitzHugh noticed that whatever incoming current intensity, the $n+h$ quantity remains close to 0.8 as shown on figures 1 and 2 from Jeff Moehlis ${ }^{1}$. We now have a system with only two dimensions : $V$ and $n$. In the following, we will consider the functions and constants given e.g. in [5]:

$$
\begin{aligned}
& V_{N a}=115 m V ; V_{K}=-12 m V ; V_{l}=10.599 m V \\
& \bar{g}_{N a}=120 m \Omega^{-1} c m^{-2} ; \bar{g}_{K}=36 m \Omega^{-1} c^{-2} ; \bar{g}_{l}=0.3 m \Omega^{-1} c^{-2} \\
& C_{M}=1 \mu F c m^{-2} ; \bar{m}(V)=\frac{25-V}{25-V+40\left(e^{-\frac{7}{4} V+\frac{5}{2}}-e^{-\frac{1}{18} V}\right)} \\
& \alpha_{n}(V)=\frac{10-V}{100\left(e^{\frac{10-V}{10}}-1\right)} ; \beta_{n}(V)=\frac{e^{-\frac{V}{80}}}{8}
\end{aligned}
$$

\subsection{The first equation approximation}

Let $\varphi$ be the right hand side of the first equation, leaving out the intensity:

$$
\varphi:(V, n) \rightarrow-\bar{g}_{K} n^{4}\left(V-V_{K}\right)-\bar{g}_{N a} \bar{m}(V)^{3}(0.8-n)\left(V-V_{N a}\right)-\bar{g}_{l}\left(V-V_{l}\right)
$$

Figure 3 shows an example of the value of $\varphi(V, n)$ as an implicit curve $\varphi(V, n)=c$ for some constant $c$. The implicit curve representing $\varphi(V, n)=c$ (of the form $n=\delta(V)$ ) has clearly a cubic shape. FitzHugh and Nagumo then proposed to approximate $\delta$ by a degree three polynomial p (scaled and shifted to maintain its roots between 0 and 1 ).

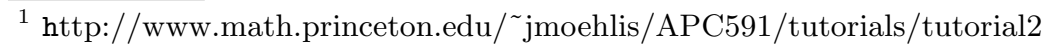




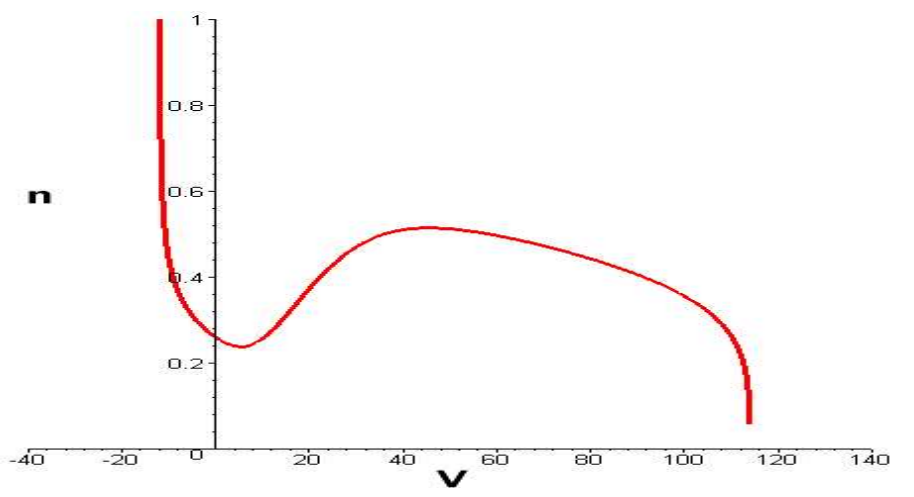

Fig. 3. Implicit representation of the first right hand side, here $c=-3$

They obtained the following equation with $w$ corresponding to a scaling and shifting of $n, v$ is a scaling of $C_{M} V$ and $I$ is modified consequently:

$$
\frac{d v}{d t}=v(1-v)(v-a)-w+I=p(v)-w+I, \text { for } 0<a<1 .
$$

Unfortunately this approximation is still too complex for a fully analytical approach. Consequently we decided to linearize $\varphi(V, n)$ by pieces and care to preserve the continuity of the curve. We also used the previous scalings and shiftings and choose to preserve $\left(v_{-}, \mathrm{p}\left(v_{-}\right)\right)$and $\left(v_{+}, \mathrm{p}\left(v_{+}\right)\right)$, the respective coordinates of the local minimum and maximum of $\mathrm{p}\left(v_{-}=\frac{a+1-\sqrt{a^{2}-a+1}}{3}, v_{+}=\frac{a+1+\sqrt{a^{2}-a+1}}{3}\right)$, as well as the two extreme roots. As shown on figure 4, p is now approached by

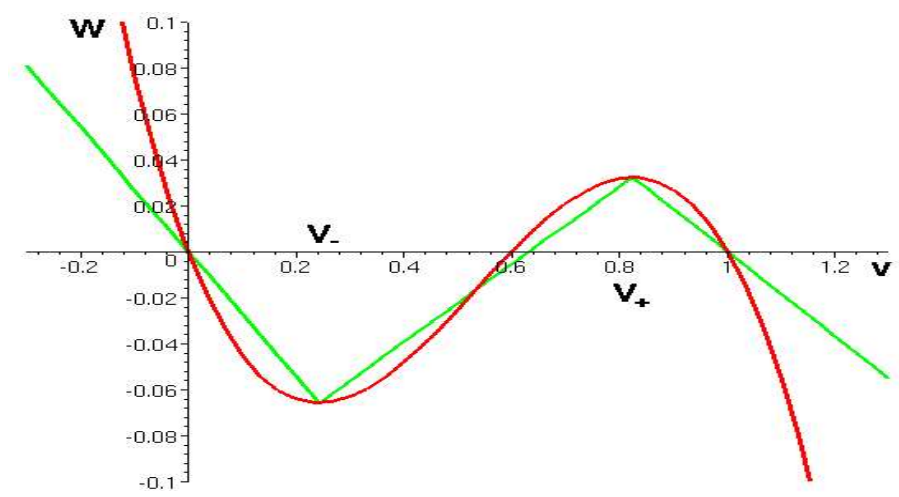

Fig. 4. The piecewise affine $\tilde{p}$ and the degree 3 polynomial approximations of the first right hand side 
the following continuous and piecewise affine function, $\tilde{\mathrm{p}}$ :

$$
\tilde{\mathrm{p}}(\mathrm{v})= \begin{cases}\frac{p\left(v_{-}\right)}{v_{-}} v & \text { if } v<v_{-} \\ {\left[\frac{p\left(v_{+}\right)-p\left(v_{-}\right)}{v_{+}-v_{-}}\right] v+\left[p\left(v_{+}\right)-\frac{p\left(v_{+}\right)-p\left(v_{-}\right)}{v_{+}-v_{-}} v_{+}\right]} & \text {if } v_{-} \leq v \leq v_{+} \\ \frac{p\left(v_{+}\right)}{1-v_{+}}(1-v) & \text { if } v>v_{+}\end{cases}
$$

Finally the first equation of the system becomes: $\frac{d v}{d t}=\tilde{\mathrm{p}}(v)-w+I$.

\subsection{The second equation approximation}

Unlike FitzHugh-Nagumo, we propose to also approach the second equation the same way. Let $\psi$ be the second right hand side, before scaling and shifting: $\psi:(V, n) \rightarrow(1-n) \alpha_{n}(v)-n \beta_{n}(v)$, for $0 \leq n \leq 1$. Figure 5 shows the implicit curve $\psi(V, n)=c$, here for $c=0$. The graph $V=\chi(n)$ of this implicit curve has a logoïde shape.

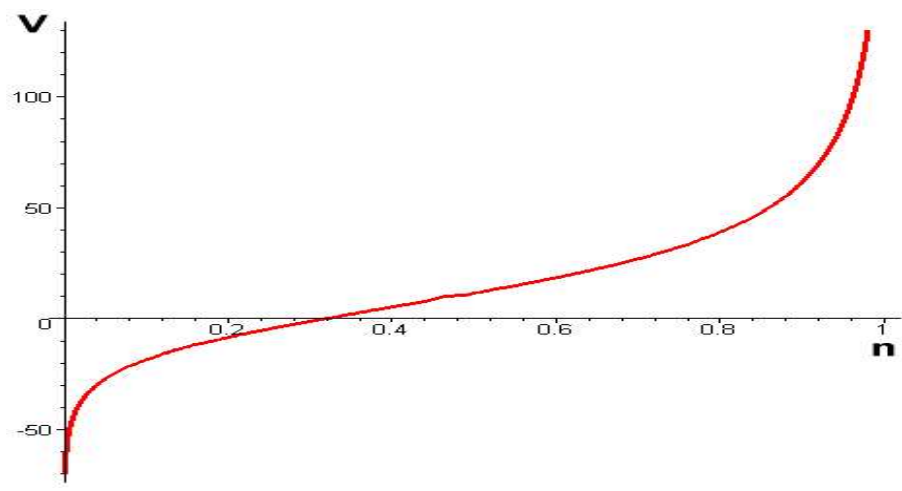

Fig. 5. Implicit representation of the second right hand side

Therefore, we linearize again by pieces and use the scales and shifts of the first approximation. This introduces extra constant factors in this second equation, so that $\psi(V, n)$ is replaced by $b v-\tilde{\chi}(w)$, with $\tilde{\chi}(w)$ the affine approximation chosen: linear for most of its range (indeed equation 1 shows that for no incoming current and with no displacement potential, the neuron must remain inactive, i.e. at $v=0$ and $I=0, \frac{d v}{d t}$ must be zero and so is $w$ ) and with a small slope 
outside two extreme thresholds $w_{0}$ and $w_{1}$, as follows:

$$
\tilde{\chi}(w)= \begin{cases}\lambda_{0}\left(w-w_{0}\right)+\gamma w_{0} & \text { if } w \leq w_{0} \\ \gamma w & \text { if } w_{0} \leq w \leq w_{1} \\ \lambda_{1}\left(w-w_{1}\right)+\gamma w_{1} & \text { if } w \geq w_{1}\end{cases}
$$

Finally the second equation of the system becomes: $\frac{d w}{d t}=b v-\tilde{\chi}(w)$.

\section{Hybrid automaton}

Now, we have a continuous and piecewise affine system modeling the neuron activity. To study the dynamics of this model (M), we consider it as a hybrid

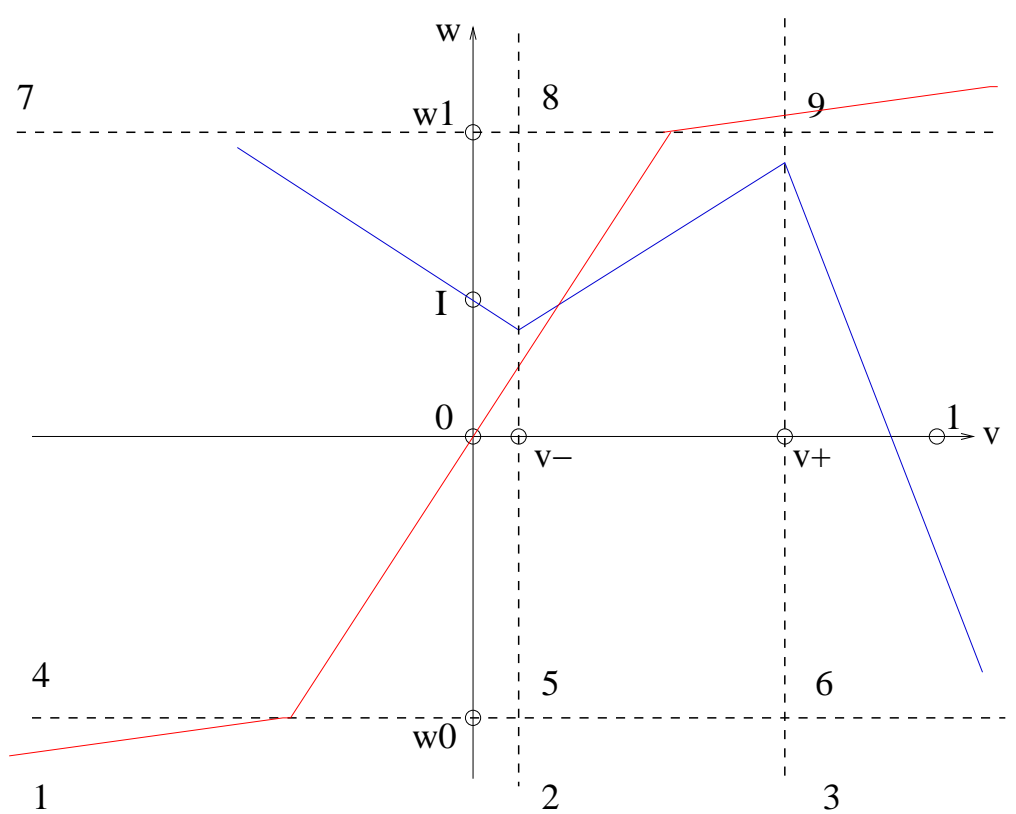

Fig. 6. Hybrid automaton of the continuous and piecewise affine model

system:

$$
\left\{\begin{array}{l}
\frac{d v}{d t}=\tilde{p}(v)-w+I \\
\frac{d w}{d t}=b v-\tilde{\chi}(w)
\end{array}\right.
$$


As there are 3 different pieces for each equation, this system is a hybrid automaton with nine contiguous states. Inside each state (rectangular cell), the trajectory evolves continuously according to its affine system (defined in sections 2.2, and 2.3). Figure 6 shows these states together with the affine system for a positive incoming current. Next section will study the different possible configurations, and therefore the transitions between the states, depending on the applied DC current I.

\section{Analysis of the (M) model dynamics}

We assume the current intensity I to be constant and positive (we are in the case where the neuron is under an external stimulation). We begin the analysis with the study of the equilibrium points of the system. This enables us to study $I$ as a bifurcation parameter of the system.

\subsection{Equilibrium points}

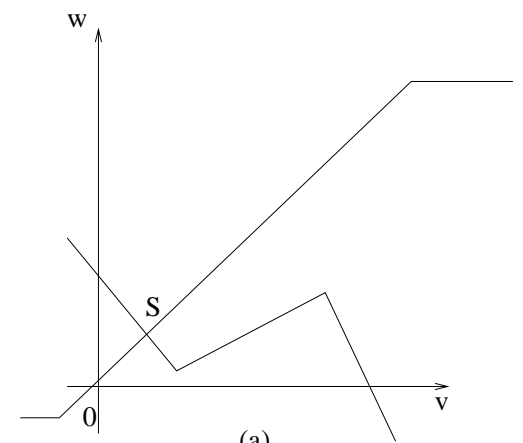

(a)

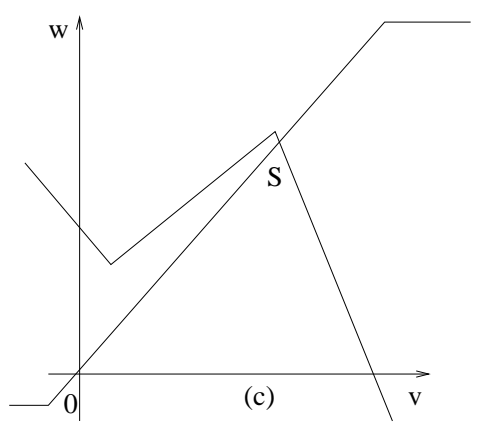

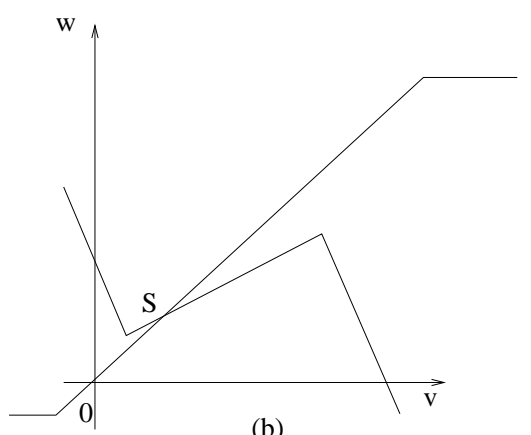

(b)

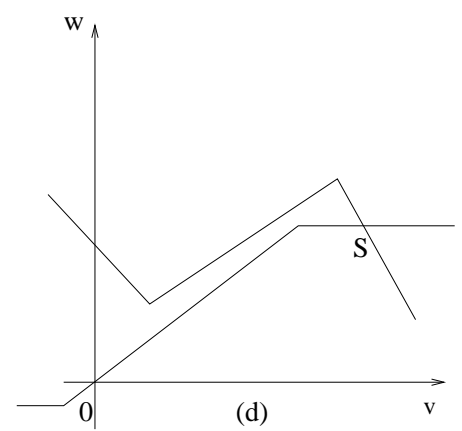

Fig. 7. Some equilibrium points of the system (M) when $I>0$ 
The equilibrium point of the system $(\mathrm{M})$ is an intersection of the two curves induced by the differential equations: $(\mathrm{w}=\tilde{\mathrm{p}}(\mathrm{v})+\mathrm{I})$ and $(\mathrm{bv}=\tilde{\chi}(w))$.

In concordance with experimental data, we assume that the linear part of $\tilde{\chi}$ is neither nearly horizontal, nor nearly vertical. Therefore, first, we assume that $\gamma$ (resp. $\lambda$ ) is small enough (resp. big enough), which means that the middle part of $\tilde{p}$ is is more gentle than the linear part of $\tilde{\chi}$ and steeper than the nearly horizontal parts of $\tilde{\chi}$. Second, as the linear part of $\tilde{\chi}$ is not extremely steep, $\frac{\gamma}{b} w_{1}>v_{+}$. Lastly, we consider that $w_{0}$ is negative so that $(v=0, w=0)$, the resting point for $I=0$, is reachable. This together gives us the following conditions:

$$
\begin{gathered}
\frac{b}{\lambda_{i}}<\frac{p\left(v_{+}\right)-p\left(v_{-}\right)}{v_{+}-v_{-}}<\frac{b}{\gamma} \\
\frac{b}{\gamma} v_{+}<w_{1} \\
w_{0}<0
\end{gathered}
$$

By looking at the original system and the graphs of the functions, we see that the experimental data of e.g. [9, Table 3], [10, §3.2.3] or [5, §2] largely fulfill these conditions. Therefore we can consider that they are non-restrictive. Consequently, we actually only have to consider the four possible configurations shown on figure 7 .

Now, we will look for equilibrium points in each cell of the automaton. This together with the stability of the points, will enable us to describe the transitions between states and the phase portrait of the approximation.

\subsection{Stability and bifurcation parameter}

The Hodgkin-Huxley system is actually parameterized by a number of parameters and shows a variety of qualitatively different behaviors depending on these parameter values. $[5,6]$, for instance, have explored numerically the dynamics of the $\mathrm{HH}$ equations for a wide range of parameter values in the multiple-parameter space, that is, they examined the global structure of bifurcations, by way of numerical simulations. In particular, they considered the externally applied DC current as the basic bifurcation parameter [5, §3.3] and used numerical simulations with the AUTO software [3] to detect the bifurcations.

Our idea in this section is to show that our linearized approximation models also this behavior. The method is to first search for equilibrium points corresponding to the system associated to the cell. For instance, if the point is stable and belongs to cell, many trajectories entering this cell will converge to this point and the automaton will remain in this state. If the point is unstable, there is a possibility of periodical behavior, etc. The trick is that the necessary conditions so that an equilibrium point is located in a certain cell, will actually induce some equivalent conditions on the incoming intensity:

First, for any intensity and under the conditions (4), (5), (6), there are no fixed point in states $1,2,3,7$ and 8 . In state 4 , the equilibrium point verifies 
the following: $w^{*}=\frac{p\left(v_{-}\right)}{v_{-}} v^{*}+I \quad ; \quad b v^{*}=\gamma w^{*}$. Then a simple computation shows that belonging to cell 4 (i.e. $v^{*}<v_{-}$and $w_{0}<w<w_{1}$ )) is equivalent to $I<I_{1}$ and $I_{0}<I<I_{1}^{\prime}$, where $I_{1}=\frac{b}{\gamma} v_{-}-p\left(v_{-}\right), I_{0}=\frac{\gamma}{b}\left[\frac{b}{\gamma}-\frac{p\left(v_{-}\right)}{v_{-}}\right] w_{0}$ and $I_{1}^{\prime}=\frac{\gamma}{b}\left[\frac{b}{\gamma}-\frac{p\left(v_{-}\right)}{v_{-}}\right] w_{1}$. Now, as $w_{0}<0, I_{0}$ is negative. Also, using condition 5 , we have $I_{1}<I_{1}^{\prime}$. Moreover, when computing the jacobian of the system at the fixed points one can show that this point is actually stable. So we have the nice condition on $I$ :

- whenever $0 \leq I<I_{1}$, there exist a stable fixed point inside cell 4 and we are in case (a).

In the same manner, we can show that there exists two other threshold intensities $I_{2}=\frac{b}{\gamma} v_{+}-p\left(v_{+}\right)$and $I_{3}=w_{1}+\frac{p\left(v_{+}\right)}{1-v_{+}}\left(\frac{\gamma}{b} w_{1}-1\right)$, so that:

- whenever $I_{1}<I<I_{2}$, there exists an unstable fixed point inside cell 5 and we are in case (b).

- whenever $I_{2}<I<I_{3}$, there exists a stable fixed point inside cell 6 and we are the case (c).

And, the last possible fixed points are located in cells 8 and 9 with the condition:

- whenever $I>I_{3}$ there exists a stable fixed point inside cell 9 and we are in case (d).

In conclusion, we analytically proved that the neuronal stimulus I (equivalently the externally applied DC current) is a bifurcation parameter of our linearized system. In the following section we actually prove that when a stable fixed point exists in a cell, the automaton tends to switch to the state containing this fixed point. Therefore the neuron emits one or more spike and then stabilizes at a given potential. On the contrary, in case (b), we will see that the configuration leads to a periodic behavior.

\subsection{Phase portrait}

The phase space associated to our model is divided into nine cells defined by the automaton (6). In the previous section, we proved that in cases (a), (c) and (d) the linearized system has only one fixed point. This point is located in a certain cell, so that whenever a trajectory arrives in this cell, it is attracted to the fixed point. Then, the question is: do we have global convergence towards the fixed point? The idea is to show that for every initial condition $\left(v_{i}, w_{i}\right)$, the associated trajectory tends to cross the cell containing the fixed point.

Well, as shown on figure 8 , the two nullclines of our system divide the plan into four parts. Each one of those four parts has then a different combination of $v$ and $w$ derivative signs. On the smae figure, we show an example trajectory simulated with CONTENT (an environment for continuation and bifurcation analysis of dynamical systems [14]). For instance, in the part defined by $\left(v^{\prime}>0\right.$ 


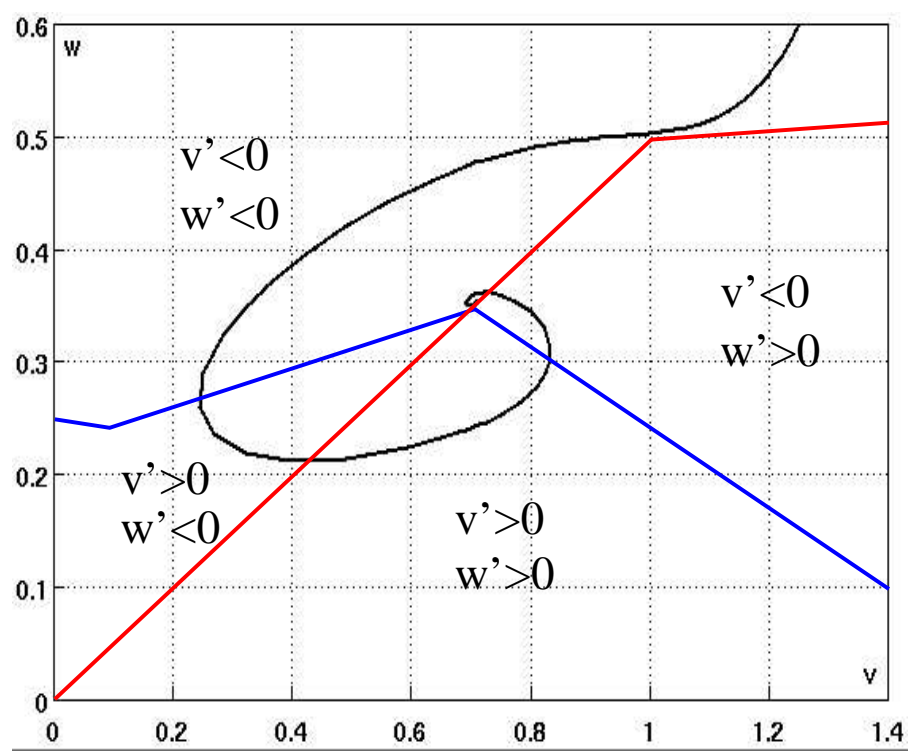

Fig. 8. Trajectory behavior using the sign of the derivatives (here for $I>0$ )

and $w^{\prime}>0$ ), the trajectory evolves in concordance, i.e. $v$ and $w$ are increasing. Now, since the third affine part of the $v$-nullcline decreases in $v$, the trajectory must cross it at some point. When this happens, $w$ keeps increasing while $v$ now starts to decrease as the trajectory is in the $\left(v^{\prime}<0\right.$ and $\left(w^{\prime}>0\right)$ part. The key point is that a trajectory will always turn anti-clockwise in the phase space. In conclusion, this together with the analysis of section 4.2 proves the following:

- whenever $0 \leq I<I_{1}$, all the trajectories collapse to the stable fixed point of cell 4 . This correspond to a spike emission followed by a return back to the resting potential.

- whenever $I_{1}<I<I_{2}$, all the trajectories keep turning around the unstable fixed point of cell 5. This correspond to oscillations of the potential.

- whenever $I_{2}<I$, all the trajectories expand to a stable fixed point inside cell 6,8 or 9 . This correspond to a stimulation by a very high incoming intensity with a spike emission and a stabilization at a high potential.

\section{Quality of the Approximation}

We now confront the theoretical results of the previous section with the numerical simulations of the Hodgkin-Huxley model. The simulations are done with CONTENT or Matlab. We first produce simulations of the original HodgkinHuxley model and compare them with simulations of our hybrid model. Then we present an alternative way of linearizing which gives an even better quality of simulations but no fully analytical analysis yet. 


\subsection{Hodgkin-Huxley model}
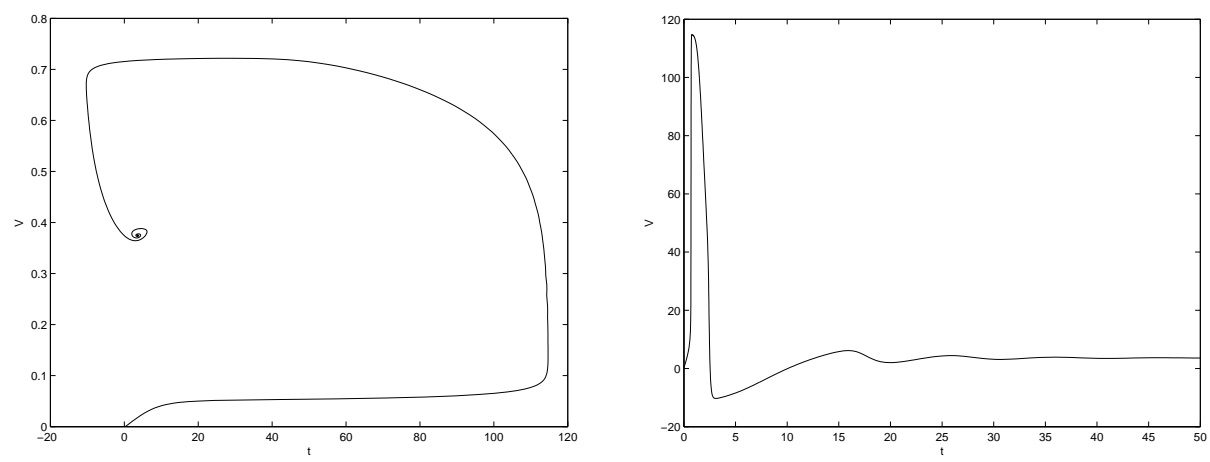

Fig. 9. Excitable state (low incoming intensity) in a simulation of Hodgkin-Huxley model: fixed point in the phase space corresponding to the Spike solution for the displacement current

The simulations of the Hodgkin-Huxley model show that there are two main characteristic for the neuronal behavior: a spike solution and a periodic solution shown respectively on figures 9 and 10. Here we used Matlab as the system is too complex for CONTENT (CONTENT had some troubles to draw the trajectory before finding the limit cycle and the trajectory even crossed itself !). Next section will produce the simulations of our linearized model for the two
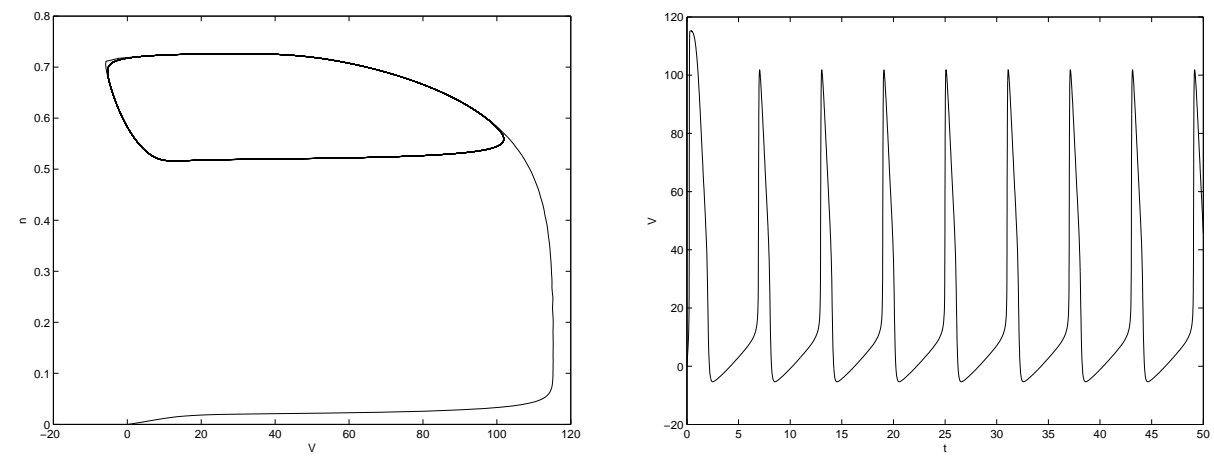

Fig. 10. Periodic state (high incoming intensity) in a simulation of Hodgkin-Huxley model: limit cycle in the phase space corresponding to Oscillations of the displacement current 
equivalent situations.

\subsection{The continuous and piecewise affine model}

The analysis of section 4 has shown that our model also produces the two characteristic behaviors: an excitable state and a periodic state. We here show the good numerical properties of these two states. Indeed, on figure 11, the left simulation is the convergence towards the stable fixed point and the right simulation shows the quality of our approximation of the spike solution. Analytically, within this excitable state (e.g. one of the configurations (a), (c) or (d) holds), the neuron emits actually a finite number of spikes, each one corresponding to a full loop around the fixed point. This does not really shows up in the Hodgkin-Huxley model as the other spikes are very small. On the contrary, in our model, the scales are much smaller and the other spikes are more visible. Moreover our
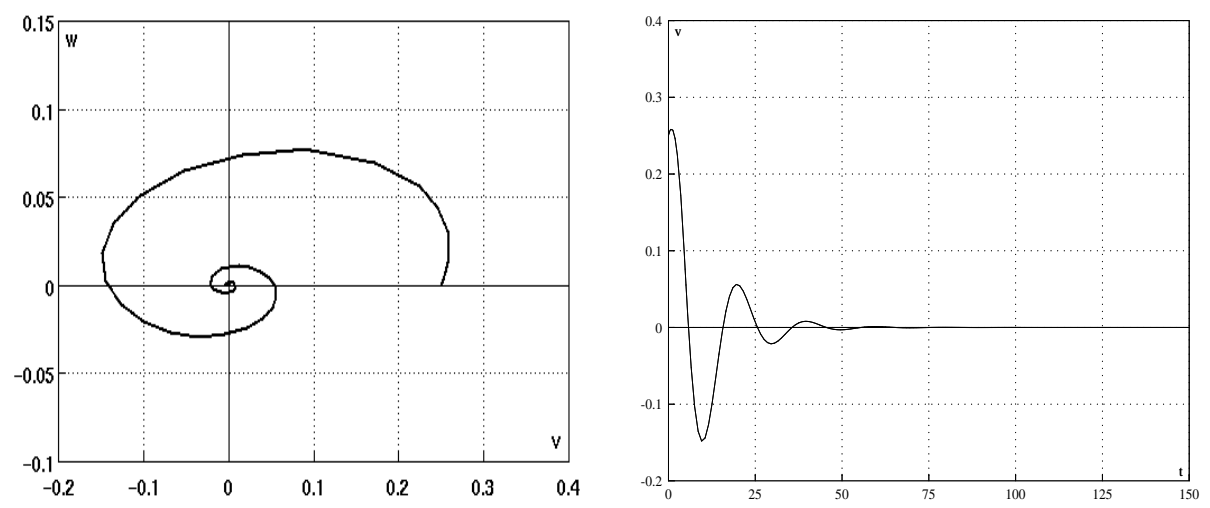

Fig. 11. Excitable state within the hybrid system

model reproduces the main property of the Hodgkin-Huxley model, that is to say the ability to generate oscillations when the current intensity is within an appropriate range. The right simulation of figure 12 shows this oscillatory state, namely the periodic state. Also, the CONTENT simulator can now detect easily the limit cycle, as shown on the left simulation on figure 12 .

When comparing with the simulations of the Hodgkin-Huxley model of the previous subsection, we clearly see the good quality of the approximation. Indeed, the continuity and the accuracy of our approximation ensures a good regularity of the new solution. 

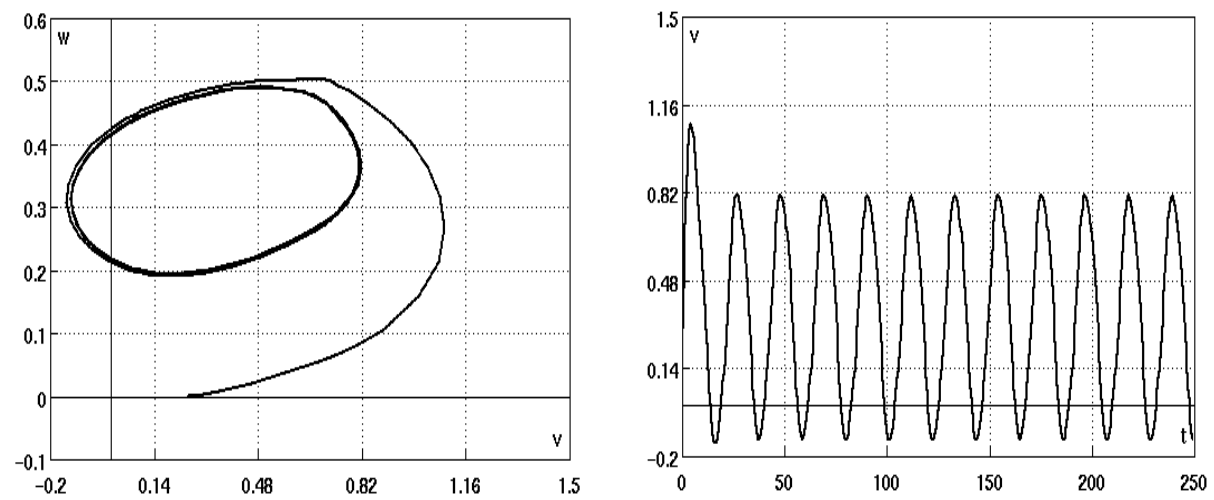

Fig. 12. Periodical state within the hybrid system

\subsection{Another linearization for an hybrid approach: multi-dimensional interpolation}

In this section we propose to show the use of multi-dimensional interpolation instead of the scheme of section 2.2 (using implicit representations). The idea is to
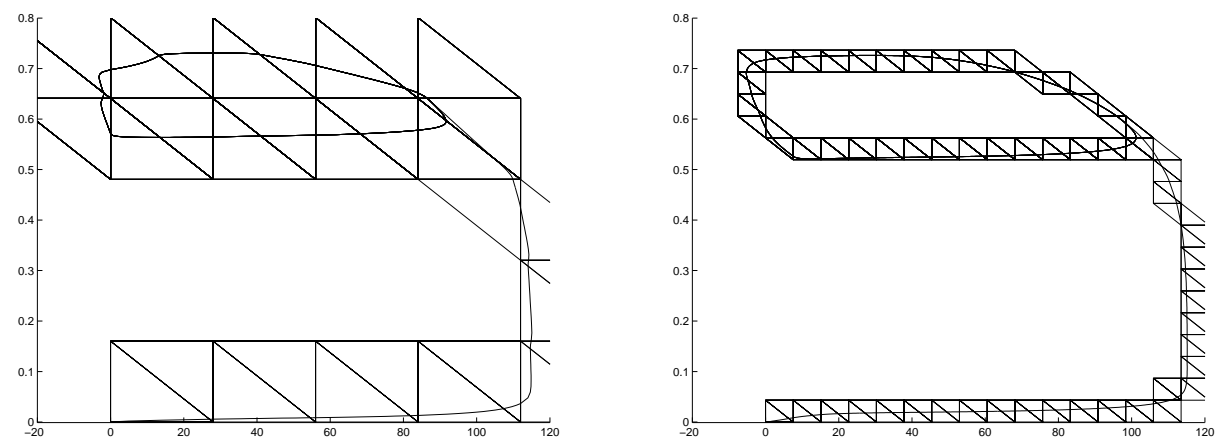

Fig. 13. Matlab Simulations of the periodic state using linear interpolation with 30 and 200 points on the mesh

build a mesh of the phase space (this can be done dynamically as the trajectories evolve). For the Hodgkin-Huxley model we can use either the 4-dimensional or the 2-dimensional version. For the sake of visualization, we produce here figures linearizing in dimension 2. The system of A. Girard [8,2] uses interpolation at each node of the mesh to define a linear approximation of the system in each cell (simplex). Figure 13, shows the periodic trajectory of figure 12 for different mesh sizes. One can check the convergence of the trajectory when the mesh is refining. 
Indeed, linearization can always be close enough to the original model so that the phase portrait of the linearization reproduces the full original behavior [8]. Also, we are able to automatically compute fixed points or stable and unstable invariants and we can semi-automatically compute e.g. the limit cycles (i.e. a point close enough to the cycle must be provided manually) or even the local attracting sets. Still, this method does not allow us to analyse any property of the system. The study of the Intensity as a bifurcation parameter, for instance, seems still pretty difficult with this approach.

\section{Conclusion}

The Hodgkin-Huxley model only considers two different ionic species $(\mathrm{K}+, \mathrm{Na}+)$ and a leakage current. However, even for this four dimensional model, the mathematical analysis is complex and most of the results are numerical. We therefore need simplified models to understand the neuronal dynamics better.

Our approach was first to reduce the dimension of the Hodgkin-Huxley model (using FitzHugh-Nagumo approach) and then to approximate each equation of the reduced system by a continuous and piecewise affine function. Indeed the continuity of our approximation ensures the regularity of the new solution and the piecewise affine approximation allows us to compute the solutions analytically. Both simulations and analytical study of our model bring to the fore the two characteristic features of the neuronal behavior:

- The spike solution

$\hookrightarrow$ correspond to the generation of a finite number of action potentials.

$\hookrightarrow$ is a transient state that characterize the property of neuronal excitability.

- The periodic solution

$\hookrightarrow$ correspond to the generation of a infinite number of action potentials.

$\hookrightarrow$ is an asymptotic state that shows the presence of a limit cycle.

We also formally proved that the applied current intensity is a bifurcation parameter of the linearized system.

With this case-study, we showed how continuous and piecewise affine hybrid systems can be used to analytically compute phase portraits that approach complex dynamical systems with no loss of intrinsic properties. Next step would then be to provide an automatic or quasi-automatic symbolic computation of the phase portrait of piecewise affine systems. Indeed, we are able to automatically compute fixed points or stable and unstable invariants. Still the full automatic analysis is not yet possible as we can only compute e.g. the limit cycles with some manual inputs. Also, a combination of different kind of linearizations would allow multi-resolution and therefore an even higher quality of simulation together with the semi-automatical analysis. 


\section{References}

1. L.F. Abbott and T. Kepler. Model neurons: From hodgkin-huxley to hopfield. In L. Garrido, editor, Statistical Mechanics of Neural Networks, pages 5-18. SpringerVerlag, Berlin, 1990.

2. Eugene Asarin, Thao Dang, and Antoine Girard. Reachability of non-linear systems using conservative approximations. In Proceedings of the 2003 Hybrid Systems: Computation and Control, Prague, The Czech Republic, April 2003.

3. E.J. Doedel, R.C. Paffenroth, A.R. Champneys, T.F. Fairgrieve, Yu.A. Kuznetsov, B. Sandstede, and X. Wang. Auto 2000: Continuation and bifurcation software for ordinary differential equations (with homcont). Technical report, California Institute of Technology, February 2001. http://auto2000. sourceforge.net.

4. Jean Della Dora, Aude Maignan, Mihaela Mirica-Ruse, and Sergio Yovine. Hybrid computation. In Bernard Mourrain, editor, Proceedings of the 2001 International Symposium on Symbolic and Algebraic Computation, London, Ontario, Canada. ACM Press, New York, July 2001.

5. Hidekazu Fukai, Shinji Doi, Taishin Nomura, and Shunsuke Sato. Hopf bifurcations in multiple-parameter space of the hodgkin-huxley equations i. global organization of bistable periodic solutions. Biological Cybernetics, 82:215-222, 2000.

6. Hidekazu Fukai, Shinji Doi, Taishin Nomura, and Shunsuke Sato. Hopf bifurcations in multiple-parameter space of the hodgkin-huxley equations ii. singularity theoretic approach and highly degenerate bifurcations. Biological Cybernetics, 82:223-229, 2000.

7. Wulfram Gerstner and Werner M. Kistler. Spiking Neuron Models: Single Neurons, Populations, Plasticity. Cambridge University Press, August 2002.

8. Antoine Girard. Approximate solutions of ordinary differential equations using piecewise linear vector fields. In Proceedings of the 2002 Computer Algebra in Scientific Computing, Big Yalta, Ukraine. Springer-Verlag, September 2002.

9. A.L. Hodgkin and A.F. Huxley. A quantitative description of membrane current and its application to conduction and excitation in nerve. Journal of Physiology, 177:500-544, 1952.

10. Frank C. Hoppensteadt. An Introduction to the Mathematics of Neurons: Modeling in the Frequency Domain. Number 14 in Cambridge Studies in Mathematical Biology. Cambridge University Press, 2 edition, 1997.

11. Frank C. Hoppensteadt and Charles S. Peskin. Modeling and Simulation in Medicine and Life Sciences. Springer-Verlag, 2 edition, 2002.

12. E.M. Izhikevich. Neural excitability, spiking, and bursting. International Journal of Bifurcation and Chaos, 10:1171-1266, 2000

13. Eugene M. Izhikevich. Resonate-and-fire neurons. Neural Networks, 14(6-7):883$894,2000$.

14. Yu.A. Kuznetsov and V.V. Levitin. Content: integrated environment for analysis of dynamical system, 1997. http://www.maths.ex.ac.uk/ hinke/courses/Content.

15. James D. Murray. Mathematical Biology. Springer-Verlag, Heidelberg, 3 edition, 1993.

16. Arnaud Tonnelier. Dynamique non-linéaire et bifurcations en neurosciences mathématiques. PhD thesis, Université Joseph Fourier, Grenoble, France, October 2001. 